December 2020, Vol. 4, $\mathrm{N}^{\circ} 1$

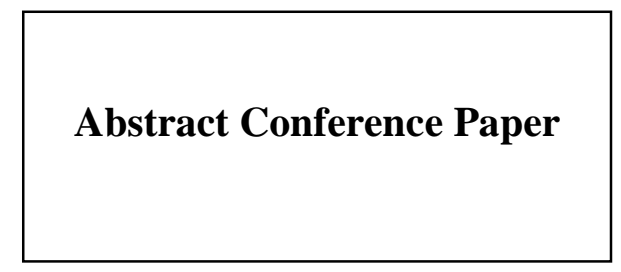

\title{
La Importancia del sector Minero para el Perú
}

\author{
Alfredo Dammert L.*1 \\ Pontificia Universidad Católica del Perú, Lima, Perú
}

El Perú es uno de los principales exportadores de minerales a nivel mundial. En el cuadro 1 se puede apreciar que es el sexto productor de oro, tercer productor de plata, segundo productor de cobre, cuarto productor de plomo y tercer productor de zinc.

\section{Cuadro 1. Principales productores de minerales}

\begin{tabular}{|l|l|}
\hline Producto & Pais\% Produccion 2016 \\
\hline Oro & $\begin{array}{l}\text { China 15\% Australia 9\% Rusia 8\% EEUU 7\% Sud Africa 5\% } \\
\text { Perú 5\% Canadá 5\% }\end{array}$ \\
\hline Plata & $\begin{array}{l}\text { México 21\% China 12\% Perú 8\% Chile 6\% Rusia 6\% Australia } \\
6 \%\end{array}$ \\
\hline Cobre & Chile 30\% Perú 9\% China 9\% EEUU 7\% Congo 5\% \\
\hline Plomo & China 46\% Australia 13\% EEUU 7\% Perú 6\% México 5\% \\
\hline $\begin{array}{l}\text { 1 E-mail } \\
\text { adammertlira@ gmail.com }\end{array}$
\end{tabular}

Dammert, A. (2020). La Importancia del sector Minero para el Perú. Journal of Economics, Finance and International Business, 4(1),XX-XX. Doi: http:// dx.doi.org/10.20511/jefib.2020 


\section{Zinc \\ China 36\% Australia 12\% Perú 11\% India 6\% EEUU 6\% \\ Carbón \\ China 45\% EEUU 13\% India 7\% Australia 5\% Indonesia 5\%}

\section{Elaboración propia}

Las exportaciones mineras peruanas representan aproximadamente el $60 \%$ de las exportaciones del país contribuyendo a la generación de divisas y permitiendo realizar importaciones que son necesarias para el crecimiento de la economía: vehículos, alimentos, artículos farmacéuticos, combustibles, y maquinaria y equipos, entre otros.

Los principales productos minero-metalúrgicos por valor de exportación son: el cobre que representa el 50\% de las exportaciones mineras, el oro que representa el $30 \%$ de las exportaciones mineras, el zinc, que representa el $7.5 \%$ de las exportaciones mineras, y el plomo que representa el $5.5 \%$ de las exportaciones mineras. Los demás productos: plata, estaño, hierro, molibdeno y otros representan en conjunto el $7 \%$ de las exportaciones mineras.

Por lo tanto, el cobre y el oro constituyen las mayores fuentes de ingresos de las exportaciones mineras para el Perú. Dada su importancia, en este artículo se analizará la evolución y perspectivas de las exportaciones de estos dos productos.

\section{Exportaciones Peruanas de Productos Mineros}

Como se puede apreciar en el Cuadro 2 el valor de exportaciones de cobre se incrementó de US\$5,835 millones en 2009 a US\$13,893 millones en 2019, o sea en $138 \%$, mientras que las exportaciones de oro lo hicieron en $25 \%$ y las exportaciones totales en el período se incrementaron en $70 \%$. Respecto al volumen de exportaciones, este se mantuvo relativamente constante para el oro (el aumento fue por el alza de cotizaciones), pero se duplicó para el cobre por la entrada de grandes proyectos a partir de 2015: Constancia en Cuzco, Las Bambas en Apurímac, y la ampliación de Cerro Verde en Arequipa.

\section{Cuadro 2. Exportaciones Peruanas de Metales US\$millones}

Fuente: Dammert, Alfredo, Economía Minera (a ser publicado en 2021)

\begin{tabular}{|l|l|l|l|l|l|l|l|l|l|l|}
\hline Año & Cobre & Oro & Zinc & Plata & Plomo & Estaño & Hierro & Molibdeno & Otros & Total \\
\hline 2009 & 5,935 & 6,791 & 1,233 & 214 & 1,116 & 591 & 298 & 276 & 27 & 16,482 \\
\hline 2010 & 8,879 & 7,745 & 1,696 & 118 & 1,579 & 842 & 523 & 492 & 29 & 21,903 \\
\hline 2011 & 10,721 & 10,235 & 1,523 & 219 & 2,427 & 776 & 1,030 & 564 & 31 & 27,526 \\
\hline 2012 & 10,731 & 10,746 & 1,352 & 210 & 2,575 & 558 & 845 & 428 & 22 & 27,467 \\
\hline 2013 & 9,821 & 8,536 & 1,414 & 479 & 1,776 & 528 & 857 & 356 & 23 & 23,789 \\
\hline 2014 & 8,875 & 6,729 & 1,504 & 331 & 1,523 & 540 & 647 & 360 & 38 & 20,545 \\
\hline
\end{tabular}




\begin{tabular}{|l|l|l|l|l|l|l|l|l|l|l|}
\hline 2015 & 8,175 & 6,537 & 1,507 & 138 & 1,542 & 342 & 350 & 220 & 27 & 18,836 \\
\hline 2016 & 10,171 & 6,610 & 1,465 & 120 & 1,658 & 344 & 344 & 280 & 30 & 21,001 \\
\hline 2017 & 13,773 & 7,979 & 2,376 & 118 & 1,707 & 370 & 427 & 363 & 44 & 27,157 \\
\hline 2018 & 14,925 & 8,239 & 2,563 & 123 & 1,530 & 335 & 486 & 612 & 11 & 28,824 \\
\hline 2019 & 13,893 & 8,482 & 2,103 & 76 & 1,530 & 371 & 979 & 638 & 2 & 28,074 \\
\hline
\end{tabular}

De lo expuesto se puede apreciar que la minería ha tenido un desarrollo poco dinámico en los últimos 10 años excepto en el caso del cobre. Sin embargo, el incremento de precios (Cuadro 3) ha contribuido significativamente al aumento de ingresos por exportaciones.

Para poder apreciar el incremento de los precios de los metales se analizará la dinámica de los mercados de cobre y oro y sus perspectivas.

\section{Cuadro 3. Cotizaciones de Principales Metales}

Fuente: Dammert, Alfredo, Economía Minera, op.cit.

\section{Los Mercados Mundiales de Cobre y Oro}

Se puede observar en el Cuadro 4 que el consumo mundial de cobre ha tenido un crecimiento de $2.5 \%$ en promedio por año. Este crecimiento ha variado en cada década: en la década del 90 que fue un período de bonanza mundial, el consumo de cobre creció a 3.5\% por año. En cambio, entre 2000 y 2017, en que la economía mundial enfrentó varios problemas, el consumo creció sólo al $2.6 \%$ por año. Por otra parte, la participación de los países ha variado significativamente. Hasta el 2000, Estados Unidos fue el principal consumidor mundial. A partir de dicho año, China tuvo un crecimiento vertiginoso pasando de consumir algo más de 500 mil TM en 1990, a más de 11 millones de TM en 2017, con lo cual su participación en el consumo mundial pasó de $4.8 \%$ del total a $50.2 \%$. En el futuro se espera que el principal mercado de este metal sea la China y otros países asiáticos.

\section{Cuadro 4. Evolución del Consumo de Cobre Refinado 1980, 1990 y 2008-2017 (Millones T.)}

Fuente: Dammert, Alfredo, op. cit. y World Bureau of Metal Statistics Reino Unido) (varios años)

\begin{tabular}{|c|c|c|c|c|c|c|c|c|c|c|c|c|}
\hline & 1980 & 1990 & 2008 & 2009 & 2010 & 2011 & 2012 & 2013 & 2014 & 2015 & 2016 & 2017 \\
\hline \multicolumn{13}{|l|}{ Europa } \\
\hline Alemania & & & 2,407 & 1,134 & 1,312 & 1,247 & 1,110 & 1,120 & 1,162 & 1,219 & 1,243 & 1,176 \\
\hline Italia & & & 635 & 523 & 619 & 608 & 570 & 552 & 625 & 613 & 596 & 633 \\
\hline Rusia & 1,300 & 1,000 & 717 & 410 & 457 & 587 & 652 & 665 & 611 & 330 & 371 & 304 \\
\hline
\end{tabular}




\begin{tabular}{|c|c|c|c|c|c|c|c|c|c|c|c|c|}
\hline \multirow{3}{*}{$\begin{array}{l}\text { Total } \\
\text { Europa }\end{array}$} & \multicolumn{2}{|l|}{ Año } & Cobre & \multirow{2}{*}{\multicolumn{2}{|c|}{\begin{tabular}{c|c}
\multicolumn{2}{|c}{ Oro } \\
, 568 & 3,970
\end{tabular}}} & \multicolumn{2}{|c|}{ Zine } & \multicolumn{2}{|c|}{ Plata } & \multicolumn{2}{|c|}{ Plomo } & Estaño \\
\hline & 4,816 & 4,17 & \begin{tabular}{l|l}
3 & 4,625 \\
\end{tabular} & & & 3,979 & 3,763 & $\mid$\begin{tabular}{||l}
3,737 \\
\end{tabular} & 3,878 & 3,710 & 3,78 & \begin{tabular}{|l|l|}
1 & 3,689 \\
\end{tabular} \\
\hline & & & & & & & & & & & & \\
\hline $\begin{array}{l}\text { Total } \\
\text { Africa }\end{array}$ & & & US $A 2 B 6$ & $210 \mathrm{~s} \$$ & $/$ peq4 & $236 \mathrm{sc} / \mathrm{lb}$ & b 244 & $1193 / \mathrm{oz}$ & 137 & $\mathrm{U} / \mathrm{1} / \mathrm{d} / \mathrm{6}$ & 140 & US $\mathbf{k} 1 \mathbf{b} 9$ \\
\hline Asia & & & & & & & & & & & & \\
\hline China & 370 & 512 & 5,150 & 7,086 & 7,385 & 7,881 & 8,896 & $9,830^{\circ}$ & 11,30 & \begin{tabular}{|l|l|l|}
3 & 11,353
\end{tabular} & 311,6 & \begin{tabular}{|l|l|}
42 & 11,790 \\
\end{tabular} \\
\hline Japón & \begin{tabular}{|c|}
1,158 \\
2005 \\
\end{tabular} & 1,57 & \begin{tabular}{|c|c|}
7 & 1,184 \\
& 167 \\
\end{tabular} & 875 & $\begin{array}{l}1,060 \\
445\end{array}$ & 1,003 & $\begin{array}{l}985 \\
\$ 3\end{array}$ & 996 7.3 & 1,072 & $\begin{array}{r}998 \\
44\end{array}$ & 973 & $\begin{array}{c}998 \\
361\end{array}$ \\
\hline Corea d\&l & & & 815 & 933 & 856 & 184 & 721 & 722 & 759 & 705 & 759 & 656 \\
\hline & 2006 & & 305 & & 604 & & 10 & & & & & \\
\hline Taiwán & 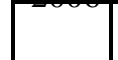 & & 582 & 494 & 532 & 457 & 432 & 438 & 465 & 471 & 507 & 498 \\
\hline Total Asia & 2007 & & 9,912 & 11,629 & \begin{tabular}{|l|l|}
912,202 \\
695
\end{tabular} & 2,413 & $\begin{array}{l}3 \mid \mathbf{1 3}, 322 \\
147\end{array}$ & $\begin{array}{r}14,231 \\
13 .\end{array}$ & $|15,83|$ & $\begin{array}{r}7 \mathbf{1 6 , 0 2 0} \\
118\end{array}$ & 16,3 & \begin{tabular}{|c|c|}
98 & $\mathbf{1 6 , 5 6 4}$ \\
\end{tabular} \\
\hline América & & & & & & & & & & & & \\
\hline Brasil & 2008 & & 3805 & 328 & 458 & 400 & $\$ 432$ & \begin{tabular}{||ll|l}
395 & 15.
\end{tabular} & 384 & 43495 & 511 & 5885 \\
\hline México & & & 299 & 284 & 274 & 30 & 322 & 334 & 361 & 393 & 423 & 512 \\
\hline EEUU & 1,888 & 2,15 & \begin{tabular}{l|l}
0 & 2,0007
\end{tabular} & 1,637 & 1,754 & 11,745 & P & $1,826^{4}$. & 1,767 & 1,798 & 1,81 & \begin{tabular}{l|l}
1 & 1,6475
\end{tabular} \\
\hline & 2010 & & 3,0988 & 2,584 & 2,832 & 2,817 & 2,860 & $2,909$. & 2,871 & 2,960 & 3,0 & \begin{tabular}{l|l}
6 & 3,042
\end{tabular} \\
\hline America & & & & & & & & & & & & \\
\hline Total & 2011 & & 1540 & 130 & 1471 & 20 & 9929 & $62 \quad 35$. & 36 & $27^{109}$ & 23 & $49^{1216}$ \\
\hline Oceanía & 2012 & & 361 & & 668 & & $\$ 8$ & 31. & & 94 & & 990 \\
\hline $\mid \begin{array}{l}\text { TOTAL } \\
\text { MUNDI }\end{array}$ & $\begin{array}{l}9,364 \\
2013\end{array}$ & $\theta$ & $\begin{array}{c}\mathbf{4 8 , 1 4 :} \\
332\end{array}$ & $10 \mathrm{~d} 116$ & $\begin{array}{l}19,3 \\
410\end{array}$ & -566 & $\$ 7$ & $\begin{array}{r}21,133 \\
23 .\end{array}$ & 322,7 & 22,893 & 23, & $\begin{array}{r}23,464 \\
1041\end{array}$ \\
\hline & 2014 & & 311 & & 1266 & & 98 & 19. & .1 & 95 & & 1023 \\
\hline & 2015 & & 250 & & 1160 & & 88 & 15. & & 81 & & 730 \\
\hline & 2016 & & 221 & & 1248 & & 95 & 17 & & 85 & & 816 \\
\hline & 2017 & & 280 & & 1257 & & 131 & 17 & 7 & 105 & & 912 \\
\hline
\end{tabular}




\begin{tabular}{|l|l|l|l|l|l|l|}
\hline 2018 & 296 & 1269 & 133 & 15.7 & 102 & 915 \\
\hline 2019 & 273 & 1392 & 116 & 16.2 & 91 & 846 \\
\hline
\end{tabular}

El mercado del oro, por ser un metal precioso, depende de factores diferentes a los del cobre. El Cuadro 5 muestra que el consumo de este producto depende por un lado directamente de la actividad económica, como es principalmente su consumo para fabricación de joyas, y por otro lado depende de la situación económica y financiera mundial: estabilidad mundial, riesgos, alternativas de inversión, etc. En este último caso que la compra de activos de inversión: barras, monedas, ETFs, y compras por parte de bancos centrales, ha tenido grandes fluctuaciones.

Cuadro 5. Oferta y Demanda del Oro a Nivel Mundial (Toneladas Métricas) 


\begin{tabular}{|c|c|c|c|c|c|c|c|c|}
\hline & 2010 & 2011 & 2012 & 2013 & 2014 & 2015 & 2016 & 2017 \\
\hline \multicolumn{9}{|l|}{ PRODUCCIÓN } \\
\hline Mina & 2743.3 & 2844 & 2916.6 & 3071.6 & 3137.8 & 3208.1 & \multicolumn{2}{|c|}{\begin{tabular}{|l|l|}
3285.1 & 3312.4 \\
\end{tabular}} \\
\hline Cobertura & \begin{tabular}{|l|}
-108.8 \\
\end{tabular} & 22.5 & -45.3 & -27.9 & 104.9 & 12.9 & 32.7 & -27.6 \\
\hline Reciclado & 1680.3 & 1655.8 & 1677.2 & 1256.2 & 1195.1 & 1130.2 & 1292.4 & 1168.2 \\
\hline $\begin{array}{l}\text { Total } \\
\text { Producción }\end{array}$ & 4314.8 & 4522.3 & 4548.5 & 4299.9 & 4437.8 & 4351.2 & 4610.2 & 4453 \\
\hline CONSUMO & & & & & & & & \\
\hline Fabricación & 2502.2 & 2515.1 & 2515.3 & 3083 & 2881.3 & 2801.8 & 2330.8 & 2577.3 \\
\hline Joyas & 2041.7 & 2086.5 & 2134 & 2727.1 & 2532.6 & 2469.8 & 2007.4 & 2244.2 \\
\hline Técnologías & 460.5 & 428.6 & 381.3 & 355.9 & 348.7 & 332 & 323.4 & 333.1 \\
\hline $\begin{array}{l}\text { Barras- } \\
\text { monedas }\end{array}$ & 1204.2 & 1502.1 & 1311.1 & 1729.6 & 1065.2 & 1090.1 & 1071.3 & 1043.3 \\
\hline ETFs & 429.6 & 323 & 306.1 & -912.3 & -184.2 & -125.1 & 547 & 206.4 \\
\hline Banco Central & 79.2 & 480.8 & 569.3 & 623.8 & 583.9 & 576.5 & 389.8 & 374.8 \\
\hline $\begin{array}{l}\text { Total } \\
\text { Demanda }\end{array}$ & 4215.1 & 4730 & 4701.8 & 4524.2 & 4346.2 & 4343.3 & 4338.2 & 4201.8 \\
\hline Precio \$/oz tr & 1224.52 & 1571.52 & 1668.98 & 1411.23 & 1266.4 & 1160.06 & 1250.8 & 1257.15 \\
\hline
\end{tabular}

Fuente: Dammert, Alfredo, op. cit. y Gold Council.

\section{Tendencias y Perspectivas}

En este artículo se ha tratado sobre la importancia de la minería para el Perú. Los dos principales metales para la economía peruana son el cobre y el oro pues representan la mayor parte de las exportaciones mineras. Por lo tanto, la pregunta es cuál es el futuro de la minería peruana y cuáles son los temas principales que se deben considerar.

Se ha visto que el crecimiento de las exportaciones mineras peruanas ha sido importante. Sin embargo, en los últimos 10 años, si bien la producción de cobre ha aumentado, la producción de oro se ha mantenido estable. Por otro lado, los precios se han incrementado contribuyendo al crecimiento de las exportaciones. A su vez, el consumo de cobre se ha elevado considerablemente en China y países asiáticos. 
Respecto al cobre, es importante continuar con el desarrollo de nuevos proyectos ya que el Perú es uno de los países con mayor potencial de proyectos mineros. Por ello es aconsejable que el gobierno se preocupe por facilitar su desarrollo, el cual se ha visto frenado por problemas sociales que no se han manejado adecuadamente. Por otra parte, dado que el principal mercado del cobre es la China, es recomendable que nuestro país mantenga buenas relaciones comerciales con éste. Además, es de esperar que una parte de inversiones importantes en el sector provenga de dicho país.

En cuanto al oro, el Perú tiene buenas perspectivas para expandir sus exportaciones. A la fecha existen algunos proyectos a nivel mundial, aunque son pocos y en el pasado, como en el caso del cobre, los problemas sociales entorpecieron su desarrollo en el Perú. En cuanto a mercados, como se ha visto, el mercado del oro es fluctuante, aunque el potencial de nueva oferta es limitado. Por lo tanto, si el Perú desarrollara nuevos proyectos mineros de oro, contaría con oportunidades de mercado para colocar sus productos.

Palabras claves: Sector Minero; Tendencias; Mercados globales

JEL Code: L72; Q33 